\title{
ON INVARIANTS OF DISCRETE SERIES REPRESENTATIONS OF CLASSICAL P-ADIC GROUPS
}

\author{
MARKo TADIĆ
}

July 4, 2010.

\begin{abstract}
To an irreducible square integrable representation $\pi$ of a classical $p$-adic group, C. Mœglin has attached invariants $\operatorname{Jord}(\pi), \pi_{c u s p}$ and $\epsilon_{\pi}$. These triples classify square integrable representations modulo cuspidal data (assuming a natural hypothesis). The definition of these invariants in $[\mathrm{M}]$ is rather simple - in terms of induced representations, except at one case when a coherent normalization of standard intertwining operators is required. In this paper we show how one can define this case also in terms of induced representations.
\end{abstract}

\section{INTRODUCTION}

To an irreducible square integrable representation $\pi$ of a classical $p$-adic group, C. Mœglin has attached a triple

$$
\left(\operatorname{Jord}(\pi), \epsilon_{\pi}, \pi_{\text {cusp }}\right) .
$$

Such a triple satisfies certain requirements. Triples satisfying that requirements are called admissible triples (see the end of the introduction, or $[\mathrm{M}]$, or $[\mathrm{MT}]$ ). Under a natural hypothesis (called the basic assumption), admissible triples classify irreducible square integrable representations of a classical $p$-adic group, modulo cuspidal data.

The above invariants are very natural and important from the point of view of the representation theory (in particular, Jordan blocks Jord $(\pi)$ are very important for the theory of $R$-groups and understanding of non-unitary duals). They are equally important and natural from the point of view of theory of automorphic forms (in particular of local Langlands correspondences).

The definition of these invariants is remarkable simple in almost all cases. This does not mean that finding of these invariants is simple (particularly hard is finding of Jordan blocks for given $\pi$ ).

We shall briefly explain the definition of these invariants. For simplicity, in this introduction we shall deal with symplectic and split special odd orthogonal groups (our paper deals with more general classical groups; see the following section). The group of (split) rank $n$ from one of these series will be denoted by $S_{n}$ (we shall fix one series of these groups). More details regarding notation in this case can be found in [T1].

A non-archimedean local field $F$ will be fixed. We shall denote by ||$_{F}$ the normalized absolute value on $F$. The character of $G L(p, F)$, obtained by composition of ||$_{F}$ with the determinant homomorphism, will be denoted also by ||$_{F}$.

Let $\rho$ be an irreducible cuspidal representation of $G L(p, F)$ and let $n$ be a positive integer (all representations considered in this paper will be smooth, and moreover admissible). Denote

$$
\left[\rho,||_{F}^{n} \rho\right]:=\left\{\rho,\left.\left|\mathbb{1}_{F} \rho,\right|\right|_{F} ^{2} \rho, \ldots,||_{F}^{n} \rho\right\} .
$$

1991 Mathematics Subject Classification. Primary 22E50, 22 E55.

The author was partly supported by Croatian Ministry of Science, Education and Sports grant \#0370372794-2804. 
Then $\left[\rho,||_{F}^{n} \rho\right]$ is called a segment in cuspidal representations (of general linear groups). Consider the parabolically induced representation

$$
\operatorname{Ind}^{G L(n p, F)}\left(||_{F}^{n} \rho \otimes||_{F}^{n-1} \rho \otimes \cdots \otimes||_{F} \rho \otimes \rho\right),
$$

induced from a suitable parabolic subgroup, which is standard with respect to the minimal parabolic subgroup of all upper triangular matrices in the group (the parabolic induction that we consider in this paper is normalized, i.e. carries unitarizable representations to the unitarizable ones). The above induced representation contains a unique irreducible subrepresentation, which we denote by

$$
\delta\left(\left[\rho,||_{F}^{n} \rho\right]\right) .
$$

The above representation is essentially square integrable. Denote

$$
\delta(\rho, n):=\delta\left(\left[||_{F}^{-\frac{n-1}{2}} \rho,||_{F}^{\frac{n-1}{2}} \rho\right]\right) .
$$

Let $\pi$ be an irreducible square integrable representation of $S_{q}$. We shall now consider the parabolically induced representation

$$
\operatorname{Ind}^{S_{n p+q}}(\delta(\rho, n) \otimes \pi),
$$

induced from a suitable parabolic subgroup, which is again standard with respect to the minimal parabolic subgroup of all upper triangular matrices in the group.

1.1. Definition. Let $\pi$ be an irreducible square integrable representation of $S_{q}$. Jord $(\pi)$ can be defined as a set of all pairs $(\rho, n)$ as above such that

(1) $\rho$ is selfdual, i.e. $\rho$ is isomorphic to its own contragredient representation $\tilde{\rho}$;

(2) $\operatorname{Ind}^{S_{n p+q}}(\delta(\rho, n) \otimes \pi)$ is irreducible;

(3) $\operatorname{Ind}^{S_{(n+2 k) p+q}}(\delta(\rho, n+2 k) \otimes \pi)$ is reducible for some positive integer $k$.

1.2. Remarks. (i) We can define the Jordan blocks of $\pi$ in a slightly different way. From this definition is more clear precise meaning (and importance) of the Jordan blocks for the representation theory (and $R$-groups). Here we shall assume a natural hypothesis (BA) from [MT] (see the the end of the introduction). Theory of $R$-groups reduces the question of understanding of tempered representations of $S_{q}$ 's to the question of reducibility of representations $\operatorname{Ind}^{S_{n p+q}}(\delta(\rho, n) \otimes \pi)$. If $\rho$ is not selfdual, then we always have irreducibility. Suppose that $\rho$ is selfdual. Then for one parity of $n$ 's (even or odd; this should depend only on $\rho$ and series of the classical groups that we consider), $\operatorname{Ind}^{S_{n+q}}(\delta(\rho, n) \otimes \pi)$ is always irreducible, and for the other parity this representation is reducible with finitely many exceptions. Denote this exceptions by

$$
\operatorname{Jord}_{\rho}(\pi)
$$

So, for understanding tempered representations one needs to know parities and exceptions. Now $\operatorname{Jord}(\pi)$ is the union of all $\{\rho\} \times \operatorname{Jord}_{\rho}(\pi)$., when $\rho$ runs over all selfdual irreducible square integrable representations of general linear groups. 
(ii) The parity for which we can have reducibility in (i) is the following (if we assume basic assumption):

- even, if $\operatorname{Ind}^{S_{p}}\left(||_{F}^{\frac{1}{2}} \rho \otimes \mathbf{1}_{S_{0}}\right)$ reduces;

- odd, if $\operatorname{Ind}^{S_{p}}\left(||_{F}^{\frac{1}{2}} \rho \otimes \mathbf{1}_{S_{0}}\right)$ is irreducible.

1.3. Definition. Let $\pi$ be an irreducible square integrable representation of $S_{q}$. The partial cuspidal support $\pi_{\text {cusp }}$ of $\pi$ is defined to be an irreducible cuspidal representation of some $S_{q^{\prime}}$ such that there exists a representation $\tau$ of $G L\left(q-q^{\prime}, F\right)$ such that

$$
\pi \hookrightarrow \operatorname{Ind}^{S_{q}}\left(\tau \otimes \pi_{c u s p}\right)
$$

The invariant $\epsilon_{\pi}$ is called partially defined function. Partly, it is related to a parameterization of some tempered representations (and characters of $R$-groups, which are $\mathbb{Z} / 2 \mathbb{Z}$ modules in this case; such characters is enough to define on a bases, and they take values \pm 1 ). On the Galois side, $\epsilon_{\pi}$ is (partly) related to the characters of the component groups. Since the classification in $[\mathrm{M}]$ and $[\mathrm{MT}]$ is only modulo cuspidal data, only a part of the character of the component group shows up in the classification (a part will come also from the corresponding character of the partial cuspidal support). We shall not describe here how partially defined function is defined exactly (one can see [M] or [MT] or [T4] for this), but we shall present definitions which determine completely this function (one gets the rest from certain multiplicativety of these functions).

Recall that the partially defined function takes values in $\{ \pm 1\}$.

1.4. Definition. Let $\pi$ be as above.

(1) Let $a_{-}<a$ be neighbor elements in $\operatorname{Jord}_{\rho}(\pi)$. Then $\epsilon_{\pi}\left(\left(\rho, a_{-}\right)\right) \epsilon_{\pi}((\rho, a))^{-1}$ is always defined. It is equal to 1 if and only if there exists a representation $\pi^{\prime}$ of $S_{q-\left(a-a_{-}\right) p / 2}$ such that

$$
\pi \hookrightarrow \operatorname{Ind}^{S_{q}}\left(\delta\left(\left[||_{F}^{\frac{a_{-}+1}{2}} \rho,||_{F}^{\frac{a-1}{2}} \rho\right]\right) \otimes \pi^{\prime}\right)
$$

(2) Let $\operatorname{Jord}_{\rho}(\pi) \neq \emptyset$ consists of even numbers. Denote $a_{\rho, \min }=\min \left(\operatorname{Jord}_{\rho}(\pi)\right)$. Then $\epsilon_{\pi}\left(\left(\rho, a_{\rho, \min }\right)\right)$ is defined. It is equal to 1 if and only if there exists a representation $\pi^{\prime}$ of $S_{q-\frac{a_{\rho, \min }}{2}}$ such that

$$
\pi \hookrightarrow \operatorname{Ind}^{S_{q}}\left(\delta\left(\left[||_{F}^{\frac{1}{2}} \rho,||_{F}^{\frac{a_{\rho, \min }-1}{2}} \rho\right]\right) \otimes \pi^{\prime}\right)
$$

Up to now, all the definitions of invariants were quite simple (which does not mean that computing of them is simple). There is only one additional case to define, to have completely determined partially defined function. This is the case when $\operatorname{Jord}_{\rho}(\pi)$ is non-empty, consists of integers of odd parity and $\operatorname{Jord}_{\rho}\left(\pi_{\text {cusp }}\right)=\emptyset$ (the last condition is equivalent to the fact that $\operatorname{Ind}^{S_{p+q^{\prime}}}\left(\rho \otimes \pi_{\text {cusp }}\right)$ reduces $)$. In this case, it is enough to define $\epsilon_{\pi}((\rho, a))$ for some $a \in \operatorname{Jord}_{\rho}(\pi)$. As we have already mentioned, C. Møglin used suitable normalized standard intertwining operators to define $\epsilon_{\pi}((\rho, a))$. Her definition involves much more 
subtle theory then the all other (previous) cases of definition (in particular, it involves standard intertwining operators, their analytic continuation, (coherent) normalization using $L$-functions and $\epsilon$-factors, realizations of families of induced representations etc.).

It is natural to ask if we can find another definition in this case, which would be in the spirit of the previous definitions of the invariants - simply using the basic language of induced representations.

Let $\sigma$ be an irreducible cuspidal representation of $S_{q^{\prime}}$ and $\rho$ selfdual irreducible cuspidal representation of $G L(p, F)$, such that $\operatorname{Ind}^{S_{p+q^{\prime}}}(\rho \otimes \pi)$ reduces. Then it reduces into two nonequivalent irreducible pieces. Denote them by $\tau_{1}^{(\rho, \sigma)}$ and $\tau_{-1}^{(\rho, \sigma)}$. J.-L. Waldspurger noted that the normalization of standard intertwining operators that uses Mœglin in her definition of $\epsilon_{\pi}((\rho, a))$ is determined by the choice of the signs that one attaches to the irreducible pieces. Natural question is, do we need to pass through complicated procedure of normalization determined by the above choices, to define partially defined function in this case, or can we proceed directly. In this paper we give affirmative answer to this question. We prove the following result, that we can now take for the remaining part of Definition 1.4.

1.5. Proposition (continuation of Definition 1.4). Let $\pi$ be as in the definition 1.4.

(3) Suppose that $\operatorname{Jord}_{\rho}(\pi)$ is non-empty, consists of odd integers, and that $\operatorname{Ind}^{S_{p+q}}(\rho \otimes$ $\pi_{\text {cusp }}$ ) reduces (equivalently $\left.\operatorname{Jord}_{\rho}\left(\pi_{\text {cusp }}\right)=\emptyset\right)$. Denote $a_{\rho, \max }=\max \left(\operatorname{Jord}_{\rho}(\pi)\right)$. Then $\epsilon_{\pi}\left(\left(\rho, a_{\rho, \max }\right)\right)$ is defined. It is equal to 1 if and only if there exists a representation $\tau^{\prime}$ of a general linear group, such that

$$
\pi \hookrightarrow \operatorname{Ind}^{S_{q}}\left(\tau^{\prime} \otimes \delta\left(\left[||_{F} \rho,||_{F}^{\frac{a_{\rho, \max }-1}{2}} \rho\right]\right) \otimes \tau_{1}^{\left(\rho, \pi_{c u s p}\right)}\right) .
$$

The classification in [MT] is modulo basic assumption. We shall recall of the basic assumption in this case.

1.6. Basic assumption. Let $\rho$ be an irreducible selfdual cuspidal representation of $G L(p, F)$ and let $\sigma$ be an irreducible cuspidal representation of $S_{q}$. Then $\nu^{\alpha} \rho \rtimes \sigma$ reduces for some $\alpha \geq 0$. From [S] follows that such $\alpha$ is unique, and we denote it by $\alpha(\rho, \sigma)$. Shahidi proved that $\alpha(\rho, \sigma) \in\{0, \pm 1 / 2, \pm 1\}$ if $\sigma$ is generic. In particular, $\alpha\left(\rho, \mathbf{1}_{S_{0}}\right) \in\{0, \pm 1 / 2, \pm 1\}$ (here $\mathbf{1}_{S_{0}}$ denotes the trivial representation of $S_{0}$ ). Now basic assumption in this case is equivalent to the following claim: for all $\rho$ and $\sigma$ as above holds

$$
\alpha(\rho, \sigma)-\alpha\left(\rho, \mathbf{1}_{S_{0}}\right) \in \mathbb{Z} .
$$

For the basic assumption in the general case see the following section.

1.7. Admissibility condition. Let $\pi$ be an irreducible square integrable representation of $S_{q}$. Denote $\operatorname{Jord}_{0}:=\operatorname{Jord}(\pi)$. Consider all the $(k+1)$-tuples $\left(\operatorname{Jord}_{0}, \operatorname{Jord}_{1}, \ldots\right.$ $\left.\ldots, \operatorname{Jord}_{k}\right)$ of sets $(k \geq 0)$, such that for each $1 \leq i \leq k$ holds:

(1) $\operatorname{Jord}_{i} \subset \operatorname{Jord}_{i-1}$;

(2) $\operatorname{card}\left(\operatorname{Jord}_{i-1} \backslash \operatorname{Jord}_{i}\right)=2$, and if we write $\operatorname{Jord}_{i-1} \backslash \operatorname{Jord}_{i}=\left\{(\rho, a),\left(\rho^{\prime}, a^{\prime}\right)\right\}$, then $\rho \cong \rho^{\prime}$

(3) $(\rho, b) \notin \operatorname{Jord}_{i-1}$ for all $b$ satisfying $\min \left(a, a^{\prime}\right)<b<\max \left(a, a^{\prime}\right)$;

(4) $\epsilon_{\pi}((\rho, a))=\epsilon_{\pi}\left(\left(\rho, a^{\prime}\right)\right)$. 
Let $\left(\operatorname{Jord}_{0}, \operatorname{Jord}_{1}, \ldots, \operatorname{Jord}_{k_{\max }}\right)$ be a $(k+1)$-tuples as above, of maximal length. Then the admissibility condition can be expressed in the following way: for any selfdual irreducible cuspidal representation $\rho$ of a general linear group, such that $\operatorname{Jord}_{\rho}(\pi) \neq \emptyset$, if we denote $J_{\rho}^{\max }=\left\{b ;(\rho, b) \in \operatorname{Jord}_{k_{\max }}\right\}$, then holds

- $\operatorname{card}\left(J_{\rho}^{\max }\right)=\alpha\left(\rho, \pi_{\text {cusp }}\right)$ if $\alpha\left(\rho, \pi_{\text {cusp }}\right) \in \mathbb{Z}$;

- $\operatorname{card}\left(J_{\rho}^{\max }\right)=\alpha\left(\rho, \pi_{\text {cusp }}\right) \pm \frac{1}{2}$ if $\alpha\left(\rho, \pi_{\text {cusp }}\right) \in\left(\frac{1}{2}+\mathbb{Z}\right)$, and then we have

$$
\epsilon_{\pi}\left(\left(\rho, \min \left(J_{\rho}^{\max }\right)\right)\right)=\left\{\begin{aligned}
1 & \text { if } \operatorname{card}\left(J_{\rho}^{\max }\right)=\alpha\left(\rho, \pi_{\text {cusp }}\right)+\frac{1}{2} \\
-1 & \text { if } \operatorname{card}\left(J_{\rho}^{\max }\right)=\alpha\left(\rho, \pi_{\text {cusp }}\right)-\frac{1}{2}
\end{aligned}\right.
$$

At the end of the introduction, we shall describe briefly the content of the paper. In the next section we introduce notation and recall of some definitions. The third section deals with the proof of the above proposition in a simple case, while the fourth section uses this simple case to prove the proposition in the general case.

We are thankful to C. Mœglin for discussions on the topic of the paper, and for reading of the paper and remarks. Conversations with C. Jantzen, A. Moy and G. Muić motivated writing of this paper.

\section{Notation}

In this section we shall very briefly recall of the notation for classical groups and general linear groups that we use in the paper. Detailed description of notation can be found in [MT] and [Z] (see also [T1] for connected split classical groups).

Fix a local non-archimedean field $F$. In the paper we shall consider symplectic, orthogonal and unitary groups. If we are working with the unitary groups, then $F^{\prime}$ will denote a separable quadratic extension of $F$ which enters the definition of the unitary groups. Otherwise, $F^{\prime}$ denotes $F$. If $F^{\prime} \neq F$, then $\theta$ denotes the non-trivial $F$-automorphism of $F^{\prime}$. Otherwise, $\theta$ denotes the identity mapping on $F$. The modulus character of $F^{\prime}$ is denoted by ||$_{F^{\prime}}$. The character $|\operatorname{det}|_{F^{\prime}}$ of $G L\left(n, F^{\prime}\right)$ will be denoted by $\nu$.

Denote by $G$ the group of rational points of a reductive group defined over $F$. We shall consider in the paper only smooth representations of such groups. The Grothendieck group of the category $\operatorname{Alg}_{\text {f.l. }}(G)$ of all the representations of $G$ of finite length is denoted by $\mathfrak{R}(G)$. It carries a natural ordering $\leq$. We denote by s.s. $(\tau)$ the semi simplification of $\tau \in \operatorname{Alg}_{\text {f.l. }}(G)$. For $\pi_{1}, \pi_{2} \in \operatorname{Alg}_{\text {f.l. }}(G)$, the fact s.s. $\left(\pi_{1}\right) \leq$ s.s. $\left(\pi_{2}\right)$ we write shorter $\pi_{1} \leq \pi_{2}$.

We shall now recall of the notation for the general linear groups mainly $[\mathrm{Z}]$.

Let $0 \leq k \leq n$. There exists a unique standard parabolic subgroup $P_{(k, n-k)}=$ $M_{(k, n-k)} N_{(k, n-k)}$ of $G L\left(n, F^{\prime}\right)$ whose Levi factor $M_{(k, n-k)}$ is naturally isomorphic to $G L\left(k, F^{\prime}\right) \times G L\left(n-k, F^{\prime}\right)$ (standard parabolic subgroups are subgroups that contain the subgroup the of upper triangular matrices in $\left.G L\left(n, F^{\prime}\right)\right)$. For representations $\pi_{1} \in$ $\operatorname{Alg}_{\text {f.l. }}\left(G L\left(k, F^{\prime}\right)\right)$ and $\pi_{2} \in \operatorname{Alg}_{\text {f.l. }}\left(G L\left(n_{i}, F^{\prime}\right)\right), \pi_{1} \times \pi_{2} \in \operatorname{Alg}_{\text {f.l. }}\left(G L\left(n_{1}+n_{2}, F^{\prime}\right)\right)$ is defined to be the representation parabolically induced representation by $\pi_{1} \otimes \pi_{2}$ from $P_{(k, n-k)}$. Let $R=\oplus_{n \geq 0} \mathfrak{R}\left(G L\left(n, F^{\prime}\right)\right)$. Now $\times$ defines in a natural way a $\mathbb{Z}$-bilinear mapping $R \times R \rightarrow R$, which will be also denoted by $\times$. Further, $\times: R \times R \rightarrow R$ factors through $R \otimes R$ by a map which will be denoted by $m: R \otimes R \rightarrow R$. 
The normalized Jacquet module of $\pi \in \operatorname{Alg}_{\text {f.l. }}\left(G L\left(n, F^{\prime}\right)\right)$ with respect to $P_{(k, n-k)}$ is denoted by $r_{(k, n-k)}(\pi)$. The comultiplication $m^{*}(\pi)$ of $\pi$ is defined by

$$
m^{*}(\pi)=\sum_{k=0}^{n} \text { s.s. }\left(r_{(k, n-k)}(\pi)\right) \in R \otimes R .
$$

One extends $m^{*}$ additively to a mapping $m^{*}: R \rightarrow R \otimes R$ in a natural way. With $m$ and $m^{*}, R$ is a graded Hopf algebra.

For an irreducible cuspidal representation $\rho$ of a general linear group over $F^{\prime}$, and $n \in \mathbb{Z}_{\geq 0},\left[\rho, \nu^{n} \rho\right]$ denotes the set $\left\{\rho, \nu \rho, \ldots, \nu^{n} \rho\right\}$ (called a segment in the irreducible cuspidal representations of the general linear groups). The representation $\nu^{n} \rho \times \nu^{n-1} \rho \times$ $\ldots \times \nu \rho \times \rho$ contains a unique irreducible subrepresentation, denoted by $\delta\left(\left[\rho, \nu^{n} \rho\right]\right)$. These representations are essentially square integrable. We denote $\left.\delta(\rho, a)=\left[\nu^{-\frac{a-1}{2}} \rho, \nu^{\frac{a-1}{2}} \rho\right]\right)$ for $a \in \mathbb{Z}_{\geq 0}$.

For an irreducible essentially square integrable representation $\delta$ of $G L\left(n, F^{\prime}\right)$, we can find unique $e(\delta) \in \mathbb{R}$ and irreducible unitarizable square integrable representation $\delta^{u}$ such that $\delta=\nu^{e(\delta)} \delta^{u}$.

Now we recall of the notation for the classical $p$-adic groups following $[\mathrm{MT}]$ (see also [MVW] and [T1]). We shall fix one of the following series $\left\{S_{n}\right\}_{n}$ of classical groups over $F$.

The symplectic group $S p(2 n, F)$ is denoted by $S_{n}$. We have here the Witt tower; we denote by $V_{n}$ the symplectic space of dimension $2 n$ in this tower (we denote $V_{0}$ also by $\left.Y_{0}\right)$.

Consider now the case of odd orthogonal groups. We fix an anisotropic orthogonal vector space $Y_{0}$ over $F$ of odd dimension (1 or 3 ), and consider the Witt tower based on $Y_{0}$. For each $n$ satisfying $2 n+1 \geq \operatorname{dim} Y_{0}$, we have exactly one space $V_{n}$ in the tower of dimension is $2 n+1$. The special orthogonal group of this space is denoted by $S_{n}$.

In the case of even-orthogonal groups, we fix an anisotropic orthogonal space $Y_{0}$ over $F$ of even dimension, and consider the Witt tower based on $Y_{0}$. For $n$ satisfying $2 n \geq \operatorname{dim}_{F}\left(Y_{0}\right)$, there is exactly one $V_{n}$ in the tower of dimension $2 n$. The orthogonal group of $V_{n}$ is denoted by $S_{n}$.

At the end, consider the case of unitary groups $U\left(n, F^{\prime} / F\right)$. Here $F^{\prime}$ denotes a separable quadratic extension of $F$. We have also an anisotropic unitary space $Y_{0}$ over $F^{\prime}$, and the Witt tower of unitary spaces $V_{n}$ based on $Y_{0}$.

If $\operatorname{dim}_{F^{\prime}}\left(Y_{0}\right)$ is odd (i.e. 1), then for each $n$ satisfying $2 n+1 \geq \operatorname{dim}_{F^{\prime}}\left(Y_{0}\right)$, there exists the unique $V_{n}$ in the tower of dimension $2 n+1$. The unitary group of this space is denoted by $S_{n}$.

If $\operatorname{dim}_{F^{\prime}}\left(Y_{0}\right)$ is even (i.e. 0$)$, then for each $n$ satisfying $2 n \geq \operatorname{dim}_{F^{\prime}}\left(Y_{0}\right)$, take the unique $V_{n}$ in the tower having dimension $2 n$. Denote its unitary group by $S_{n}$.

Minimal parabolic subgroup in $S_{n}$ will be fixed (only standard parabolic subgroups with respect to the fixed minimal parabolic subgroup will be considered in the paper). Convenient matrix realizations of the groups $S_{n}$ and the description of their standard parabolic subgroups for $S p(2 n, F)$ and split $S O(n+1, F)$ can be found in [T1].

Fix one of the series $\left\{S_{n}\right\}_{n}$ as above. Let $n^{\prime}$ be the Witt index of $V_{n}\left(n^{\prime}=n-\frac{1}{2} \operatorname{dim}_{F^{\prime}}\left(Y_{0}\right)\right.$ if $V_{n}$ is symplectic or even-orthogonal or even-unitary group, and otherwise $n^{\prime}=n-$ 
$\frac{1}{2}\left(\operatorname{dim}_{F^{\prime}}\left(Y_{0}\right)-1\right)$. Take $k^{\prime}$ satisfying $0 \leq k \leq n^{\prime}$. Then there exists a standard parabolic subgroup $P_{(k)}=M_{(k)} N_{(k)}$ of $S_{n}$, whose Levi subgroup $M_{(k)}$ is naturally isomorphic to $G L\left(k, F^{\prime}\right) \times S_{n-k}$ (the series of symplectic and split orthogonal groups are considered in [T1] and $[\mathrm{B}]$, while in the case of other series of groups the isomorphism is defined analogously). Group $P_{(k)}$ is the stabilizer of an isotropic space of dimension $k$. For $\pi \in \operatorname{Alg}_{\text {f.l. }}\left(G L\left(k, F^{\prime}\right)\right)$ and $\sigma \in \operatorname{Alg}_{\text {f.l. }}\left(S_{n-k}\right)$, the representation parabolically induced by $\pi \otimes \sigma$ is denoted by

$\pi \rtimes \sigma$.

We shall often use that $\pi_{1} \rtimes\left(\pi_{2} \rtimes \sigma\right) \cong\left(\pi_{1} \times \pi_{2}\right) \rtimes \sigma$. For $\pi$ as above, denote by $\check{\pi}$

$$
g \longmapsto \tilde{\pi}(\theta(g)),
$$

where $\tilde{\pi}$ is the contragredient representation of $\pi$. Then s.s. $(\pi \rtimes \sigma)=$ s.s. $(\check{\pi} \rtimes \sigma)$. Note that if $\pi \rtimes \sigma$ is irreducible, then $\pi \rtimes \sigma \cong \check{\pi} \rtimes \sigma$. We say that a representation $\pi$ of a general linear group over $F^{\prime}$ is $F^{\prime} / F$-selfdual if $\pi \cong \check{\pi}$. If $F^{\prime}=F$, then we shall say also simply that $\pi$ is selfdual.

The normalized Jacquet module of $\tau \in \operatorname{Alg}_{\text {f.l. }}\left(S_{n}\right)$ with respect to $P_{(k)}$ is denoted by $s_{(k)}(\tau)$. Let

$$
R(S)=\underset{n}{\oplus} \mathfrak{R}\left(S_{n}\right) .
$$

The above sum runs over all integers $n \geq \frac{1}{2}\left(\operatorname{dim}_{F^{\prime}}\left(Y_{0}\right)-1\right)$ if we consider odd-orthogonal or odd-unitary groups, and otherwise over all $\left.n \geq \frac{1}{2} \operatorname{dim}_{F^{\prime}}\left(Y_{0}\right)\right)$.

Now $\rtimes$ induces in a natural way a mapping $R \times R(S) \rightarrow R(S)$, which is denoted again by $\rtimes$. For $\tau \in \operatorname{Alg}_{\text {f.l. }}\left(S_{n}\right)$, denote

$$
\mu^{*}(\tau)=\sum_{k=0}^{n^{\prime}} \text { s.s. }\left(s_{(k)}(\tau)\right)
$$

where $n^{\prime}$ denotes the Witt index of $V_{n}$. Extend $\mu^{*}$ additively to $\mu^{*}: R(S) \rightarrow R \otimes R(S)$. Denote

$$
M^{*}=(m \otimes 1) \circ\left(` \otimes m^{*}\right) \circ \kappa \circ m^{*}: R \rightarrow R \otimes R,
$$

where ${ }^{\sim}: R \rightarrow R$ is a group homomorphism determined by the requirement that $\pi \mapsto \check{\pi}$ for all irreducible $\pi$, and $\kappa: R \times R \rightarrow R \times R$ maps $\sum x_{i} \otimes y_{i}$ to $\sum y_{i} \otimes x_{i}$. The action $\rtimes$ of $R \otimes R$ on $R \otimes R(S)$ is defined in a natural way. Then holds

$$
\mu^{*}(\pi \rtimes \sigma)=M^{*}(\pi) \rtimes \mu^{*}(\sigma)
$$

for $\pi \in R$ and $\sigma \in R(S)$.

Let $\rho$ be an irreducible $F^{\prime} / F$-selfdual cuspidal representation of a general linear group. Suppose that $x, y \in \mathbb{R}$ satisfy $y-x \in \mathbb{Z}_{\geq 0}$. Then one easily gets

$$
M^{*}\left(\delta\left(\left[\nu^{x} \rho, \nu^{y} \rho\right]\right)\right)=\sum_{i=x-1}^{y} \sum_{j=i}^{y} \delta\left(\left[\nu^{-i} \rho, \nu^{-x} \rho\right]\right) \times \delta\left(\left[\nu^{j+1} \rho, \nu^{y} \rho\right]\right) \otimes \delta\left(\left[\nu^{i+1} \rho, \nu^{j} \rho\right]\right),
$$


where $y-i, y-j \in \mathbb{Z}_{\geq 0}$ in the above sums.

Let $k \in \mathbb{Z}_{>0}$. If we are considering the symplectic series of groups (resp. one of orthogonal series of groups), then $R_{k}$ will denote the representation of $G L(k, \mathbb{C})$ on $\wedge^{2} \mathbb{C}^{k}$ $\left(\right.$ resp. Sym $\left.^{2} \mathbb{C}^{k}\right)$.

Suppose that we are considering a series of unitary groups. Then $L$-group of $G L\left(k, F^{\prime}\right)$, considered as $F$-group, is isomorphic to a semidirect product $(G L(k, \mathbb{C}) \times G L(k, \mathbb{C}))$ 人 $\operatorname{Gal}\left(F^{\prime} / F\right)$. The non-trivial element of $\theta$ of $\operatorname{Gal}\left(F^{\prime} / F\right)$ acts on the normal subgroup $G L\left(d_{\rho}, \mathbb{C}\right) \times G L\left(d_{\rho}, \mathbb{C}\right)$ by $\theta\left(g_{1}, g_{2}, 1\right) \theta^{-1}=\left({ }^{t} g_{2}^{-1},{ }^{t} g_{1}^{-1}, 1\right)$, where ${ }^{t} g$ denotes the transposed matrix of $g$. Let $\eta \in\{ \pm 1\}$, and let $R_{k}^{(\eta)}$ be the representation of the above $L$-group (of $G L\left(k, F^{\prime}\right)$ ) on $\operatorname{End}_{\mathbb{C}}\left(\mathbb{C}^{k}\right)$, defined by: $\left(g_{1}, g_{2}, 1\right) u=g_{1} u^{t} g_{2}$ and $(1,1, \theta) u=\eta^{t} u$. If $S_{n}$ is a series of groups such that dimensions of $V_{n}$ are even, then we denote by $R_{k}$ the representation $R_{k}^{(1)}$. In the case of odd dimensions, $R_{k}$ denotes $R_{k}^{(-1)}$.

Now we shall recall of the definition from [M] of the Jordan blocks $\operatorname{Jord}(\pi)$ of an irreducible square integrable representation $\pi$ of $S_{n}$.

Bellow $L\left(\rho, R_{d_{\rho}}, s\right)$ denotes the $L$-function defined by F. Shahidi.

2.1. Definition. $\operatorname{Jord}(\pi)$ is the set of all pairs $(\rho, a)$ where $\rho$ is an irreducible $F^{\prime} / F$ selfdual cuspidal representation of $G L\left(d_{\rho}, F^{\prime}\right)$ and $a \in \mathbb{Z}_{>0}$, such that:

(J1) $a$ is even if $L\left(\rho, R_{d_{\rho}}, s\right)$ has a pole at $s=0$, and odd otherwise,

(J2) $\delta(\rho, a) \rtimes \pi_{0}$ is irreducible.

For an irreducible $F^{\prime} / F$-selfdual cuspidal representation of a general linear group $\rho$, denote $\operatorname{Jord}_{\rho}(\pi)=\{a ;(\rho, a) \in \operatorname{Jord}(\pi)\}$.

We shall assume in the paper that the following basic assumption holds for any irreducible cuspidal representation $\pi_{c u s p}$ of any $S_{q}$ and any irreducible $F^{\prime} / F$-selfdual cuspidal representation $\rho$ of any $G L\left(p, F^{\prime}\right)$ :

(BA) Let

$$
a_{\rho}=\left\{\begin{array}{l}
\max \operatorname{Jord}_{\rho}\left(\pi_{\text {cusp }}\right) \quad \text { if } \operatorname{Jord}_{\rho}\left(\pi_{\text {cusp }}\right) \neq \emptyset \\
0 \quad \text { if } L\left(\rho, R_{d_{\rho}}, s\right) \text { has a pole at } s=0 \text { and } \operatorname{Jord}_{\rho}\left(\pi_{\text {cusp }}\right)=\emptyset, \\
-1 \quad \text { otherwise. }
\end{array}\right.
$$

Then $\nu^{ \pm\left(a_{\rho}+1\right) / 2} \rho \rtimes \pi_{\text {cusp }}$ reduces.

Regarding remaining two invariants, the definition of the partial cuspidal support is the same in the general case as it was in Definition 1.3, except that $\tau^{\prime}$ needs to be a representation of $G L\left(q-q^{\prime}, F^{\prime}\right)$ (instead of $G L\left(q-q^{\prime}, F\right)$, as it was there). Similarly is with the definition of the partially defined function $\epsilon_{\pi}$. The only difference is that in the general case we need to work with $F^{\prime} / F$-selfdual irreducible cuspidal representations $\rho$ (instead of selfdual, as it was the case in the introduction).

\section{THE FIRST STEP}

In this section we shall assume that $\sigma$ is an irreducible cuspidal representation of a classical group $S_{q}$, and that $\rho$ is an irreducible unitarizable cuspidal representation of a general linear group such that $\rho \rtimes \sigma$ reduces (this implies that $\rho$ is $F^{\prime} / F$-selfdual). Then 
it reduces into two nonequivalent irreducible subrepresentations, which we shall denote by $\tau_{+}$and $\tau_{-}$. Thus

$$
\rho \rtimes \sigma=\tau_{+} \oplus \tau_{-} .
$$

In the moment, the choice of signs \pm is arbitrary (in general, there is no canonical way to chose it). Later in this section, we shall make our choice compatible with Mœeglin choice in $[\mathrm{M}]$.

3.1. Remark. Observe that Lemma 1.2 of [S] (see also Theorem 1.6 there), enables us to conclude from the reducibility of $\rho \rtimes \sigma$, that

$$
\nu^{\alpha} \rho \rtimes \sigma
$$

is irreducible for all $\alpha \in \mathbb{R} \backslash\{0\}$.

In this section

$$
a_{-} \text {and } a
$$

will be odd positive integers, such that $a_{-}<a$. To simplify notation in this section, we denote

$$
n=\left(a_{-}-1\right) / 2, \quad m=(a-1) / 2 .
$$

Then $n, m \in \mathbb{Z}_{\geq 0}, n<m$.

Most of the technical claims in the following lemma can be extracted from [MT], but for the sake of completeness we give proof here.

3.2. Lemma. (1) The representation

$$
\delta\left(\left[\nu^{-n} \rho, \nu^{n} \rho\right]\right) \rtimes \sigma
$$

reduces into a sum of two nonequivalent irreducible subrepresentations $T_{ \pm}$. We characterize them by the requirement

$$
\delta\left(\left[\nu \rho, \nu^{n} \rho\right]\right) \times \delta\left(\left[\nu \rho, \nu^{n} \rho\right]\right) \otimes \tau_{ \pm} \leq \mu^{*}\left(T_{ \pm}\right) .
$$

Moreover,

$$
T_{ \pm} \hookrightarrow \delta\left(\left[\nu \rho, \nu^{n} \rho\right]\right) \times \delta\left(\left[\nu \rho, \nu^{n} \rho\right]\right) \rtimes \tau_{ \pm}
$$

(2) The multiplicity of

$$
\delta\left(\left[\nu \rho, \nu^{n} \rho\right]\right) \times \delta\left(\left[\nu \rho, \nu^{m} \rho\right]\right) \otimes \tau_{ \pm}
$$

in both

$$
\mu^{*}\left(\delta\left(\left[\nu \rho, \nu^{n} \rho\right]\right) \times \delta\left(\left[\nu \rho, \nu^{n} \rho\right]\right) \times \delta\left(\left[\nu^{n+1} \rho, \nu^{m} \rho\right]\right) \times \rho \rtimes \sigma\right)
$$

and

$$
\mu^{*}\left(\delta\left(\left[\nu^{-n} \rho, \nu^{m} \rho\right]\right) \rtimes \sigma\right)
$$

is one. 
(3) The multiplicity of $\delta\left(\left[\nu^{n+1} \rho, \nu^{m} \rho\right]\right) \otimes T_{ \pm}$in

$$
\mu^{*}\left(\delta\left(\left[\nu^{-n} \rho, \nu^{m} \rho\right]\right) \rtimes \sigma\right)
$$

and in

$$
\mu^{*}\left(\delta\left(\left[\nu^{n+1} \rho, \nu^{m} \rho\right]\right) \times \delta\left(\left[\nu^{-n} \rho, \nu^{n} \rho\right]\right) \rtimes \sigma\right)
$$

is 1 .

Proof. (1) We know from Proposition 4.4 of [T2] that $\delta\left(\left[\nu^{-n} \rho, \nu^{n} \rho\right]\right) \rtimes \sigma$ reduces into a sum of two nonequivalent irreducible subrepresentations

Consider formula $(2.2)$ for $x=-n$ and $y=n$. Recall $\mu^{*}\left(\delta\left(\left[\nu^{-n} \rho, \nu^{n} \rho\right]\right) \rtimes \sigma\right)=$ $M^{*}\left(\delta\left(\left[\nu^{-n} \rho, \nu^{n} \rho\right]\right)\right) \rtimes(1 \otimes \sigma)$. To get a term of the form $* \otimes \tau_{ \pm}$in $\mu^{*}\left(\delta\left(\left[\nu^{-n} \rho, \nu^{n} \rho\right]\right) \rtimes \sigma\right)$, from $M^{*}\left(\delta\left(\left[\nu^{-n} \rho, \nu^{n} \rho\right]\right)\right)$ we must take $i=-1$ and $j=0$. The corresponding term is $\delta\left(\left[\nu \rho, \nu^{n} \rho\right]\right) \times \delta\left(\left[\nu \rho, \nu^{n} \rho\right]\right) \otimes \rho \rtimes \sigma$. This implies that terms $\delta\left(\left[\nu \rho, \nu^{n} \rho\right]\right) \times \delta\left(\left[\nu \rho, \nu^{n} \rho\right]\right) \otimes \tau_{ \pm}$ have multiplicity one in the Jacquet module of $\delta\left(\left[\nu^{-n} \rho, \nu^{n} \rho\right]\right) \rtimes \sigma$.

From Theorem 13.2 of [T2] and Remark 3.1 we get that $\delta\left(\left[\nu^{-n} \rho, \nu^{-1} \rho\right]\right) \rtimes \sigma$ is irreducible. This and a general fact that $\pi \rtimes \tau$ and $\check{\pi} \rtimes \tau$ have the same Jordan-Hölder series imply that

$$
\delta\left(\left[\nu^{-n} \rho, \nu^{-1} \rho\right]\right) \rtimes \sigma \cong \delta\left(\left[\nu \rho, \nu^{n} \rho\right]\right) \rtimes \sigma .
$$

Let $T$ be an irreducible subrepresentation of $\delta\left(\left[\nu^{-n} \rho, \nu^{n} \rho\right]\right) \rtimes \sigma$. Now we have

$$
\begin{gathered}
T \hookrightarrow \delta\left(\left[\nu^{-n} \rho, \nu^{n} \rho\right]\right) \rtimes \sigma \hookrightarrow \delta\left(\left[\rho, \nu^{n} \rho\right]\right) \times \delta\left(\left[\nu^{-n} \rho, \nu^{-1} \rho\right]\right) \rtimes \sigma \cong \\
\delta\left(\left[\rho, \nu^{n} \rho\right]\right) \times \delta\left(\left[\nu \rho, \nu^{n} \rho\right]\right) \rtimes \sigma \cong \delta\left(\left[\nu \rho, \nu^{n} \rho\right]\right) \times \delta\left(\left[\rho, \nu^{n} \rho\right]\right) \rtimes \sigma \\
\hookrightarrow \delta\left(\left[\nu \rho, \nu^{n} \rho\right]\right) \times \delta\left(\left[\nu \rho, \nu^{n} \rho\right]\right) \times \rho \rtimes \sigma
\end{gathered}
$$

(observe that above we have used isomorphism $\left.\delta\left(\left[\nu^{-n} \rho, \nu^{-1} \rho\right]\right) \rtimes \sigma \cong \delta\left(\left[\nu \rho, \nu^{n} \rho\right]\right) \rtimes \sigma\right)$. Now Frobenius reciprocity implies that corresponding Jacquet module of each $T$ (as above), must have a non trivial intertwining to $\delta\left(\left[\nu \rho, \nu^{n} \rho\right]\right) \times \delta\left(\left[\nu \rho, \nu^{n} \rho\right]\right) \otimes \rho \rtimes \sigma$. The multiplicity one that we have proved above, implies that for one $T$, we have an epimorphism from corresponding Jacquet module of $T$ onto $\delta\left(\left[\nu \rho, \nu^{n} \rho\right]\right) \times \delta\left(\left[\nu \rho, \nu^{n} \rho\right]\right) \otimes \tau_{+}$(this $T$ will be denoted by $\left.T_{+}\right)$, and for the other $T$ we have an epimorphism from the corresponding Jacquet module of $T$ onto $\delta\left(\left[\nu \rho, \nu^{n} \rho\right]\right) \times \delta\left(\left[\nu \rho, \nu^{n} \rho\right]\right) \otimes \tau_{-}$(this $T$ will be denoted by $\left.T_{-}\right)$. Frobenius reciprocity implies that $T_{ \pm} \hookrightarrow \delta\left(\left[\nu \rho, \nu^{n} \rho\right]\right) \times \delta\left(\left[\nu \rho, \nu^{n} \rho\right]\right) \rtimes \tau_{ \pm}$.

(2) We shall use now formula (2.2) for $x=-n$ and $y=m$. From this formula we get that multiplicity of representations $\delta\left(\left[\nu \rho, \nu^{n} \rho\right]\right) \times \delta\left(\left[\nu \rho, \nu^{m} \rho\right]\right) \otimes \tau_{ \pm}$are one in the corresponding Jacquet module of $\delta\left(\left[\nu^{-n} \rho, \nu^{m} \rho\right]\right) \rtimes \sigma$ (one must take the term corresponding to $i=-1, j=0)$.

In the case of $\delta\left(\left[\nu \rho, \nu^{n} \rho\right]\right) \times \delta\left(\left[\nu \rho, \nu^{n} \rho\right]\right) \times \delta\left(\left[\nu^{n+1} \rho, \nu^{m} \rho\right]\right) \times \rho \rtimes \sigma$, one first writes $\mu^{*}$ applied to this representation. Then one uses formula (2.2), considers supports, and uses the fact that $\rho$ must be on the right hand side of the tensor product $\otimes$. This gives directly the remaining multiplicity one which we needed.

(3) To get $\delta\left(\left[\nu^{n+1} \rho, \nu^{m} \rho\right]\right) \otimes T_{ \pm}$in $\mu^{*}\left(\delta\left(\left[\nu^{-n} \rho, \nu^{m} \rho\right]\right) \rtimes \sigma\right)$, from $M^{*}\left(\delta\left(\left[\nu^{-n} \rho, \nu^{m} \rho\right]\right)\right)$ we must take terms satisfying $n<-i$, which implies $i=-n-1$. From this we get that 
$j+1=n+1$. This implies the multiplicity one in this case that we needed to prove. One gets the other multiplicity one in a similar way. This completes the proof of the lemma.

Let $\pi$ be an irreducible subrepresentation of $\delta\left(\left[\nu^{-n} \rho, \nu^{m} \rho\right]\right) \rtimes \sigma$. Then we know that $\pi$ is square integrable, and

$$
\pi \hookrightarrow \delta\left(\left[\nu^{n+1} \rho, \nu^{m} \rho\right]\right) \rtimes T_{ \pm}
$$

for exactly one sign (see $[\mathrm{MT}])$. Also then $\epsilon_{\pi}\left(\left(\rho, a_{-}\right)\right)=\epsilon_{\pi}((\rho, a))$. We further know from $[\mathrm{MT}]$ that in $\delta\left(\left[\nu^{-n} \rho, \nu^{m} \rho\right]\right) \rtimes \sigma$ we have two irreducible square integrable subrepresentations, and that they are nonequivalent.

\subsection{Corollary.}

(i) Irreducible subrepresentations of $\delta\left(\left[\nu^{-n} \rho, \nu^{m} \rho\right]\right) \rtimes \sigma$ can be characterized as irreducible subquotients of $\delta\left(\left[\nu^{-n} \rho, \nu^{m} \rho\right]\right) \rtimes \sigma$ which have terms $\delta\left(\left[\nu^{n+1} \rho, \nu^{m} \rho\right]\right) \otimes T_{ \pm}$in their Jacquet modules. They will be denoted by

$$
\delta\left(\left[\nu^{-n} \rho, \nu^{m} \rho\right]_{T_{ \pm}}, \sigma\right)
$$

(ii) Then

$$
\delta\left(\left[\nu \rho, \nu^{n} \rho\right]\right) \times \delta\left(\left[\nu \rho, \nu^{m} \rho\right]\right) \otimes \tau_{ \pm} \leq \mu^{*}\left(\delta\left(\left[\nu^{-n} \rho, \nu^{m} \rho\right]_{T_{ \pm}}, \sigma\right)\right) .
$$

(iii) We can characterize $\delta\left(\left[\nu^{-n} \rho, \nu^{m} \rho\right]_{T_{ \pm}}, \sigma\right)$ as a unique irreducible subquotient of

$$
\delta\left(\left[\nu^{n+1} \rho, \nu^{m} \rho\right]\right) \times \delta\left(\left[\nu \rho, \nu^{n} \rho\right]\right) \times \delta\left(\left[\nu \rho, \nu^{n} \rho\right]\right) \times \rho \rtimes \sigma
$$

which has $\delta\left(\left[\nu \rho, \nu^{n} \rho\right]\right) \times \delta\left(\left[\nu \rho, \nu^{m} \rho\right]\right) \otimes \tau_{ \pm}$in its Jacquet module.

Proof. (i) is clear from preceding lemma.

(ii) We know that

$$
\delta\left(\left[\nu^{-n} \rho, \nu^{m} \rho\right]_{T_{ \pm}}, \sigma\right) \hookrightarrow \delta\left(\left[\nu^{n+1} \rho, \nu^{m} \rho\right]\right) \times \delta\left(\left[\nu \rho, \nu^{n} \rho\right]\right) \times \delta\left(\left[\nu \rho, \nu^{n} \rho\right]\right) \rtimes \tau_{ \pm} .
$$

From this follows that there exists a non-trivial intertwining from the Jacquet module of the left hand side to $\delta\left(\left[\nu^{n+1} \rho, \nu^{m} \rho\right]\right) \times \delta\left(\left[\nu \rho, \nu^{n} \rho\right]\right) \times \delta\left(\left[\nu \rho, \nu^{n} \rho\right]\right) \otimes \tau_{ \pm}$. The last representation is an indecomposable multiplicity one representation of length 2 , which has $\delta\left(\left[\nu \rho, \nu^{m} \rho\right]\right) \times$ $\delta\left(\left[\nu \rho, \nu^{n} \rho\right]\right) \otimes \tau_{ \pm}$for subrepresentation. This implies the statement.

(iii) Now this follows from (ii) and the above lemma.

Representation $\nu \delta \rtimes \tau_{ \pm}$has a unique irreducible subrepresentation, which we denoted by $\delta\left([\rho, \nu \rho]_{\tau_{ \pm}}, \sigma\right)$. Both these representations are subrepresentations of $\delta([\rho, \nu \rho]) \rtimes \sigma$. Therefore, $(\rho, 1),(\rho, 3) \in \operatorname{Jord}\left(\delta\left([\rho, \nu \rho]_{\tau_{ \pm}}, \sigma\right)\right)$ in both cases, and we know

$$
\epsilon_{\delta\left([\rho, \nu \rho]_{\tau_{ \pm}}, \sigma\right)}((\rho, 1))=\epsilon_{\delta\left([\rho, \nu \rho]_{\tau_{ \pm}}, \sigma\right)}((\rho, 3))
$$

from the definition of the partially defined function. Recall $\left.(\rho, 1) \notin \operatorname{Jord}_{\rho}(\sigma)\right)$ and also $\left.(\rho, k) \notin \operatorname{Jord}_{\rho}(\sigma)\right)$ for all odd integers $k \geq 3$ (since $\delta(\rho, k) \rtimes \sigma$ is always reducible). Further, 
also for even integers $k$ hold $\left.(\rho, k) \notin \operatorname{Jord}_{\rho}(\sigma)\right)$ (by definition of the parity, since we always have the irreducibility of $\delta(\rho, k) \rtimes \sigma)$.

C. Møglin has defined $\epsilon_{\delta\left([\rho, \nu \rho]_{\tau_{ \pm}}, \sigma\right)}((\rho, 1)) \in\{ \pm 1\}$ (in general, in a non-canonical way). Then $\epsilon_{\delta\left([\rho, \nu \rho]_{\tau_{+}}, \sigma\right)}((\rho, 1)) \neq \epsilon_{\delta\left([\rho, \nu \rho]_{\left.\tau_{-}, \sigma\right)}\right.}((\rho, 1))$. Therefore, we can denote irreducible subrepresentations $\tau_{ \pm}$in the decomposition of $\rho \rtimes \sigma$ in a way that holds

$$
\epsilon_{\delta\left([\rho, \nu \rho]_{\tau_{ \pm}}, \sigma\right)}((\rho, 1))= \pm 1
$$

We assume that we have made such a choice in the rest of the paper.

C. Mœglin has defined partially defined function in this case using fixed normalization of standard intertwining operators, which satisfies certain properties (see $[\mathrm{M}]$ and references there). We shall in the sequel use her normalization of the standard intertwining operators. Then $\tau_{+}$is the subrepresentation of $\rho \rtimes \sigma$ on which the normalized standard intertwining operator

$$
\nu^{s} \rho \rtimes \sigma \rightarrow \nu^{-s} \rho \rtimes \sigma
$$

acts as identity for $s=0$ (the action on $\tau_{-}$is multiplication by -1 ).

3.5. Lemma. (i) Denote

$$
\pi_{ \pm}=\delta\left(\left[\nu^{-n} \rho, \nu^{m} \rho\right]_{T_{ \pm}}, \sigma\right) .
$$

Then $\operatorname{Jord}_{\rho}\left(\pi_{ \pm}\right)=\left\{a_{-}, a\right\}$ and

$$
\epsilon_{\pi_{ \pm}}\left(\left(\rho, a_{-}\right)\right)=\epsilon_{\pi_{ \pm}}((\rho, a))= \pm 1
$$

(ii) The normalized standard intertwining operator

$$
\nu^{s} \delta\left(\rho, a_{-}\right) \rtimes \sigma \rightarrow \nu^{-s} \delta\left(\rho, a_{-}\right) \rtimes \sigma
$$

acts for $s=0$ on $T_{ \pm}$as multiplication by the scalar \pm 1 .

Proof. (i) The claim about Jordan blocks follows from Proposition 4.2 of [M]. It remains to prove the claim for the partially defined function.

Observe that

$$
\delta\left(\left[\rho, \nu^{m} \rho\right]_{\tau_{ \pm}}, \sigma\right) \hookrightarrow \delta\left(\left[\nu \rho, \nu^{m} \rho\right]\right) \rtimes \tau_{ \pm} \hookrightarrow \delta\left(\left[\nu^{2} \rho, \nu^{m} \rho\right]\right) \times \nu \rho \rtimes \tau_{ \pm} .
$$

First, this implies that $\delta\left(\left[\nu \rho, \nu^{m} \rho\right]\right) \otimes \tau_{ \pm}$is in the Jacquet module of $\delta\left(\left[\rho, \nu^{m} \rho\right]_{\tau_{ \pm}}, \sigma\right)$. One gets directly that the multiplicity of $\delta\left(\left[\nu \rho, \nu^{m} \rho\right]\right) \otimes \tau_{ \pm}$in $\mu^{*}\left(\delta\left(\left[\nu^{2} \rho, \nu^{m} \rho\right]\right) \times \nu \rho \times \rho \rtimes \sigma\right)$ is one. The multiplicity is also one in Jacquet modules of $\delta\left(\left[\nu^{2} \rho, \nu^{m} \rho\right]\right) \times \nu \rho \rtimes \tau_{ \pm}$and $\delta\left(\left[\nu^{2} \rho, \nu^{m} \rho\right]\right) \times \delta\left([\rho, \nu \rho]_{\tau_{ \pm}}, \sigma\right)$. Since $\delta\left(\left[\nu^{2} \rho, \nu^{m} \rho\right]\right) \times \delta\left([\rho, \nu \rho]_{\tau_{ \pm}}, \sigma\right)$ is a subrepresentation of $\delta\left(\left[\nu^{2} \rho, \nu^{m} \rho\right]\right) \times \nu \rho \rtimes \tau_{ \pm}$, we conclude that

$$
\delta\left(\left[\rho, \nu^{m} \rho\right]_{\tau_{ \pm}}, \sigma\right) \hookrightarrow \delta\left(\left[\nu^{2} \rho, \nu^{m} \rho\right]\right) \times \delta\left([\rho, \nu \rho]_{\tau_{ \pm}}, \sigma\right) .
$$

Denote $\pi_{ \pm}^{(k)}=\delta\left(\left[\rho, \nu^{k} \rho\right]_{\tau_{ \pm}}, \sigma\right)$ for $k \geq 1$. By 6.1 .2 of $[\mathrm{M}], \epsilon_{\pi_{ \pm}^{(m)}}((\rho, 1))$ is the scalar by which acts the normalized standard intertwining operator

$$
\delta\left(\left[\nu \rho, \nu^{m} \rho\right]\right) \times \nu^{s} \rho \rtimes \sigma \rightarrow \delta\left(\left[\nu \rho, \nu^{m} \rho\right]\right) \times \nu^{-s} \rho \rtimes \sigma
$$


for $s=0$ on the subrepresentation $\pi_{ \pm}^{(m)}$. Observe that

$$
\delta\left(\left[\nu \rho, \nu^{m} \rho\right]\right) \times \nu^{ \pm s} \rho \rtimes \sigma \hookrightarrow \delta\left(\left[\nu^{2} \rho, \nu^{m} \rho\right]\right) \times \nu \rho \times \nu^{ \pm s} \rho \rtimes \sigma .
$$

From the other side, $\epsilon_{\pi_{ \pm}^{(1)}}((\rho, 1))$ is the scalar by which acts the normalized standard intertwining operator

$$
\nu \rho \times \nu^{s} \rho \rtimes \sigma \rightarrow \nu \rho \times \nu^{-s} \rho \rtimes \sigma
$$

for $s=0$ on the subrepresentation $\pi_{ \pm}^{(1)}$. Therefore, $\epsilon_{\pi_{ \pm}^{(1)}}((\rho, 1))$ is the scalar by which acts the normalized standard intertwining operator

$$
\delta\left(\left[\nu^{2} \rho, \nu^{m} \rho\right]\right) \times \nu \rho \times \nu^{s} \rho \rtimes \sigma \hookrightarrow \delta\left(\left[\nu^{2} \rho, \nu^{m} \rho\right]\right) \times \nu \rho \times \nu^{-s} \rho \rtimes \sigma
$$

for $s=0$ on the subrepresentation $\delta\left(\left[\nu^{2} \rho, \nu^{m} \rho\right]\right) \rtimes \pi_{ \pm}^{(1)}$. Now (3.3) and (3.1) imply that the normalized standard intertwining operator action in the case (3.2) for $s=0$ on subrepresentation $\pi_{ \pm}^{(m)}$ is multiplication by $\epsilon_{\pi_{ \pm}^{(1)}}((\rho, 1))$. Therefore, $\epsilon_{\pi_{ \pm}^{(m)}}((\rho, 1))=\epsilon_{\pi_{ \pm}^{(1)}}((\rho, 1))$ $(= \pm 1)$.

First observe that from (2.2) directly follows that the multiplicity of $\delta\left(\left[\nu^{-n} \rho, \nu^{m} \rho\right]\right) \otimes \sigma$ in $\mu^{*}\left(\delta\left(\left[\nu \rho, \nu^{n} \rho\right]\right) \times \delta\left(\left[\rho, \nu^{m} \rho\right]\right) \rtimes \sigma\right)$ is 2 . Consider $\delta\left(\left[\nu^{-n} \rho, \nu^{m} \rho\right]\right) \rtimes \sigma \hookrightarrow \delta\left(\left[\rho, \nu^{m} \rho\right]\right) \times$ $\delta\left(\left[\nu^{-n} \rho, \nu^{-1} \rho\right]\right) \rtimes \sigma$. Each irreducible subrepresentation of the left hand side is also subrepresentation of the right hand side. Observe that $\delta\left(\left[\rho, \nu^{m} \rho\right]\right) \times \delta\left(\left[\nu^{-n} \rho, \nu^{-1} \rho\right]\right) \rtimes \sigma$ is isomorphic to

$$
\delta\left(\left[\rho, \nu^{m} \rho\right]\right) \times \delta\left(\left[\nu \rho, \nu^{n} \rho\right]\right) \rtimes \sigma \cong \delta\left(\left[\nu \rho, \nu^{n} \rho\right]\right) \times \delta\left(\left[\rho, \nu^{m} \rho\right]\right) \rtimes \sigma .
$$

The representation on the right hand side has two subrepresentations: $\delta\left(\left[\nu \rho, \nu^{n} \rho\right]\right) \rtimes \pi_{ \pm}^{(m)}$ (their intersection is $\{0\})$. Each of these subrepresentations has $\delta\left(\left[\nu^{-n} \rho, \nu^{m} \rho\right]\right) \otimes \sigma$ in its Jacquet module. From this follows that $\delta\left(\left[\nu^{-n} \rho, \nu^{m} \rho\right]_{T_{ \pm}} ; \sigma\right) \hookrightarrow \delta\left(\left[\nu \rho, \nu^{n} \rho\right]\right) \rtimes \pi_{ \pm}^{(m)}$ or $\delta\left(\left[\nu^{-n} \rho, \nu^{m} \rho\right]_{T_{ \pm}} ; \sigma\right) \hookrightarrow \delta\left(\left[\nu \rho, \nu^{n} \rho\right]\right) \rtimes \pi_{\mp}^{(m)}$. One easily shows that $\delta\left(\left[\nu \rho, \nu^{m} \rho\right]\right) \times$ $\delta\left(\left[\nu \rho, \nu^{n} \rho\right]\right) \otimes \tau_{ \pm}$is in the Jacquet module of $\delta\left(\left[\nu \rho, \nu^{n} \rho\right]\right) \rtimes \pi_{ \pm}^{(m)}$. Therefore

$$
\pi_{ \pm}=\delta\left(\left[\nu^{-n} \rho, \nu^{m} \rho\right]_{T_{ \pm}} ; \sigma\right) \hookrightarrow \delta\left(\left[\nu \rho, \nu^{n} \rho\right]\right) \rtimes \pi_{ \pm}^{(m)}
$$

By (i) of Proposition 6.1 of $[\mathrm{M}], \epsilon_{\pi_{ \pm}}((\rho, a))$ is the scalar by which acts the normalized standard intertwining operator

$$
\nu^{s} \delta\left(\left[\nu^{-m} \rho, \nu^{m} \rho\right]\right) \rtimes \pi_{ \pm} \rightarrow \nu^{-s} \delta\left(\left[\nu^{-m} \rho, \nu^{m} \rho\right]\right) \rtimes \pi_{ \pm}
$$

for $s=0$. Using (3.4), we get that $\epsilon_{\pi_{ \pm}}((\rho, a))$ is the scalar by which acts the normalized standard intertwining operator

$$
\nu^{s} \delta\left(\left[\nu^{-m} \rho, \nu^{m} \rho\right]\right) \times \delta\left(\left[\nu \rho, \nu^{n} \rho\right]\right) \rtimes \pi_{ \pm}^{(m)} \rightarrow \nu^{-s} \delta\left(\left[\nu^{-m} \rho, \nu^{m} \rho\right]\right) \times \delta\left(\left[\nu \rho, \nu^{n} \rho\right]\right) \rtimes \pi_{ \pm}^{(m)}
$$


for $s=0$ on the subrepresentation $\delta\left(\left[\nu^{-m} \rho, \nu^{m} \rho\right]\right) \rtimes \pi_{ \pm}$.

From the other side, again by (i) of Proposition 6.1 of $[\mathrm{M}], \epsilon_{\pi_{ \pm}^{(m)}}((\rho, a))$ is the scalar by which acts the normalized standard intertwining operator

$$
\nu^{s} \delta\left(\left[\nu^{-m} \rho, \nu^{m} \rho\right]\right) \rtimes \pi_{ \pm}^{(m)} \rightarrow \nu^{-s} \delta\left(\left[\nu^{-m} \rho, \nu^{m} \rho\right]\right) \rtimes \pi_{ \pm}^{(m)}
$$

for $s=0$. Therefore, $\epsilon_{\pi_{ \pm}^{(m)}}((\rho, a))$ is the scalar by which acts the normalized standard intertwining operator

$$
\delta\left(\left[\nu \rho, \nu^{n} \rho\right]\right) \times \nu^{s} \delta\left(\left[\nu^{-m} \rho, \nu^{m} \rho\right]\right) \rtimes \pi_{ \pm}^{(m)} \rightarrow \delta\left(\left[\nu \rho, \nu^{n} \rho\right]\right) \times \nu^{-s} \delta\left(\left[\nu^{-m} \rho, \nu^{m} \rho\right]\right) \rtimes \pi_{ \pm}^{(m)}
$$

for $s=0$, which is the same as the scalar by which acts the normalized standard intertwining operator

$$
\nu^{s} \delta\left(\left[\nu^{-m} \rho, \nu^{m} \rho\right]\right) \times \delta\left(\left[\nu \rho, \nu^{n} \rho\right]\right) \rtimes \pi_{ \pm}^{(m)} \rightarrow \nu^{-s} \delta\left(\left[\nu^{-m} \rho, \nu^{m} \rho\right]\right) \times \delta\left(\left[\nu \rho, \nu^{n} \rho\right]\right) \rtimes \pi_{ \pm}^{(m)}
$$

for $s=0$, since for $s$ in a neighborhood of 0 we have $\nu^{ \pm s} \delta\left(\left[\nu^{-m} \rho, \nu^{m} \rho\right]\right) \times \delta\left(\left[\nu \rho, \nu^{n} \rho\right]\right) \cong$ $\delta\left(\left[\nu \rho, \nu^{n} \rho\right]\right) \times \nu^{ \pm s} \delta\left(\left[\nu^{-m} \rho, \nu^{m} \rho\right]\right)$. Thus $\epsilon_{\pi_{ \pm}}((\rho, a))=\epsilon_{\pi_{ \pm}^{(m)}}((\rho, a))$.

Now we know that

$$
\epsilon_{\pi_{ \pm}}\left(\left(\rho, a_{-}\right)\right)=\epsilon_{\pi_{ \pm}}((\rho, a))=\epsilon_{\pi_{ \pm}^{(m)}}((\rho, a))=\epsilon_{\pi_{ \pm}^{(m)}}((\rho, 1))=\epsilon_{\pi_{ \pm}^{(1)}}((\rho, 1))= \pm 1
$$

The proof of (i) is now complete.

(ii) By 6.1 .1 of $[\mathrm{M}], \epsilon_{\pi_{ \pm}}((\rho, a))(= \pm 1)$ is the scalar by which acts the normalized standard intertwining operator

$$
\delta\left(\left[\nu^{n+1} \rho, \nu^{m} \rho\right]\right) \times \nu^{s} \delta(\rho, n) \rtimes \sigma \rightarrow \delta\left(\left[\nu^{n+1} \rho, \nu^{m} \rho\right]\right) \times \nu^{-s} \delta(\rho, n) \rtimes \sigma
$$

for $s=0$ on the subrepresentation $\pi_{ \pm}$. But $\pi_{ \pm}$is a subrepresentation of the representation $\delta\left(\left[\nu^{n+1} \rho, \nu^{m} \rho\right]\right) \rtimes T_{ \pm}$. And if the normalized standard intertwining operator

$$
\nu^{s} \delta(\rho, n) \rtimes \sigma \rightarrow \nu^{-s} \delta(\rho, n) \rtimes \sigma
$$

for $s=0$ acts on $T_{ \pm}$by $\lambda_{ \pm} \in\{ \pm 1\}$, then the normalized standard intertwining operator

$$
\delta\left(\left[\nu^{n+1} \rho, \nu^{m} \rho\right]\right) \times \nu^{s} \delta(\rho, n) \rtimes \sigma \rightarrow \delta\left(\left[\nu^{n+1} \rho, \nu^{m} \rho\right]\right) \times \nu^{-s} \delta(\rho, n) \rtimes \sigma
$$

for $s=0$ acts on $\delta\left(\left[\nu^{n+1} \rho, \nu^{m} \rho\right]\right) \rtimes T_{ \pm}$as $\lambda_{ \pm}$. Therefore, $\lambda_{ \pm}=\epsilon_{\pi_{ \pm}}((\rho, a))= \pm 1$. This completes the proof of (ii). 


\section{General CASe}

In this section $\pi$ will be be an irreducible square integrable representation of a classical group. Denote $\sigma=\pi_{\text {cusp }}$. Let $\rho$ an irreducible $F^{\prime} / F$-selfdual cuspidal representation of a general linear group such that $\rho \rtimes \sigma$ reduce. We decompose $\rho \rtimes \sigma=\tau_{+} \oplus \tau_{-}$in the same way as we did in the last section. We shall denote $\tau_{ \pm}$also by

$$
\tau_{ \pm 1}^{\left(\rho, \pi_{\text {cusp }}\right)}:=\tau_{ \pm}
$$

4.1. Proposition. Let $\pi$ be an irreducible square integrable representation of a classical group and let $\rho$ be an irreducible $F^{\prime} / F$-selfdual cuspidal representation of a general linear

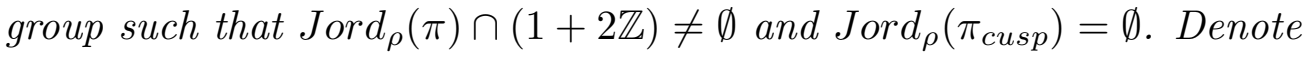

$$
a=\max \left(\operatorname{Jord}_{\rho}(\pi)\right) \text {. }
$$

Then

(1) there exists an irreducible representation $\tau$ of a general linear group such that

$$
\pi \hookrightarrow \tau \times \delta\left(\left[\nu \rho, \nu^{(a-1) / 2} \rho\right]\right) \rtimes \tau_{\epsilon_{\pi}((\rho, a))}^{\left(\rho, \pi_{\text {cusp }}\right)} ;
$$

(2) there does not exist an irreducible representation $\tau$ of a general linear group such that

$$
\pi \hookrightarrow \tau \times \delta\left(\left[\nu \rho, \nu^{(a-1) / 2} \rho\right]\right) \rtimes \tau_{-\epsilon_{\pi}((\rho, a))}^{\left(\rho, \pi_{\text {cusp }}\right)} .
$$

Proof. Recall that partially defined function $\epsilon_{\pi}$ is defined on each $\left(\rho^{\prime}, k\right) \in \operatorname{Jord}(\pi)$ for which $\rho^{\prime} \cong \rho$. We have denoted $\sigma=\pi_{\text {cusp }}$. By the definition of the admissible triple, we have in this case always

$$
\operatorname{card}\left(\operatorname{Jord}_{\rho}(\pi)\right) \in 2 \mathbb{Z},
$$

and the number of values +1 which show up as values of $\epsilon_{\pi}$ on $\operatorname{Jord}_{\rho}(\pi)$, is equal to the number of values -1 that show up as values on $\operatorname{Jord}_{\rho}(\pi)$.

Construction of square integrable representation $\pi$ in [MT] proceeds inductively. In the last step, one considers neighbor elements $b$ and $b_{-}$in $\operatorname{Jord}_{\rho}(\pi)$ on which $\epsilon_{\pi}$ is the same. In a general step, one considers $b$ and $b_{-}$which are neighbors in Jordan blocks at that level, and on which partially defined function takes the same values (Jordan blocks and partially defined functions are of the corresponding square integrable representation which shows up in that step of construction). Construction starts with strongly positive square integrable representation, which we shall denote by $\pi^{\prime}$. Condition $\operatorname{Jord}_{\rho}\left(\pi_{\text {cusp }}\right)=\emptyset$ implies that $\operatorname{Jord}_{\rho}\left(\pi^{\prime}\right)=\emptyset$. We can embed

$$
\pi^{\prime} \hookrightarrow\left(\prod_{i=1}^{\ell} \delta\left(\Gamma_{i}\right)\right) \rtimes \pi_{c u s p}
$$

(see the construction of the strongly positive representations in $[\mathrm{MT}]$ ). 
Denote by $a_{-}=\max \left\{b \in \operatorname{Jord}_{\rho}(\pi) \backslash\{a\} ; \epsilon_{\pi}((\rho, b))=\epsilon_{\pi}((\rho, a))\right\}$. Then the condition of admissibility of the triple $\left(\operatorname{Jor} d(\pi), \epsilon_{\pi}, \pi_{\text {cusp }}\right)$ implies that between $a_{-}$and $a$ must be even number of elements from $\operatorname{Jord}_{\rho}(\pi)$. Because of this, we can start the construction of $\pi$ from $\pi^{\prime}$ with the pair of Jordan blocks $\left(\rho, a_{-}\right)$and $(\rho, a)$ (observe that $a_{-}$and $a$ does not need to be neighbors in $\left.\operatorname{Jord}_{\rho}(\pi)\right)$.

Now one considers irreducible subrepresentations of $\left.\delta\left(\left[\nu^{-\left(a_{-}-1\right) / 2} \rho, \nu^{(a-1) / 2} \rho\right]\right)\right) \rtimes \pi^{\prime}$. One needs to chose irreducible subrepresentation $\pi^{(1)}$ in a way that the normalized standard intertwining operator

$$
\begin{aligned}
\left.\delta\left(\left[\nu^{\left(a_{-}+1\right) / 2} \rho, \nu^{(a-1) / 2} \rho\right]\right)\right) & \times \nu^{s} \delta\left(\rho, a_{-}\right) \rtimes \pi^{\prime} \\
& \left.\rightarrow \delta\left(\left[\nu^{\left(a_{-}+1\right) / 2} \rho, \nu^{(a-1) / 2} \rho\right]\right)\right) \times \nu^{-s} \delta\left(\rho, a_{-}\right) \rtimes \pi^{\prime}
\end{aligned}
$$

acts on $\pi^{(1)}$ by scalar $\epsilon_{\pi}((\rho, a))$ for $s=0$. Taking into account (4.1), one needs to chose irreducible subrepresentation $\pi^{(1)}$ in a way that the normalized standard intertwining operator

$$
\begin{aligned}
\left.\delta\left(\left[\nu^{\left(a_{-}+1\right) / 2} \rho, \nu^{(a-1) / 2} \rho\right]\right)\right) \times \nu^{s} \delta\left(\rho, a_{-}\right) \times\left(\prod_{i=1}^{\ell} \delta\left(\Gamma_{i}\right)\right) \rtimes \pi_{\text {cusp }} \\
\left.\rightarrow \delta\left(\left[\nu^{\left(a_{-}+1\right) / 2} \rho, \nu^{(a-1) / 2} \rho\right]\right)\right) \times \nu^{-s} \delta\left(\rho, a_{-}\right) \times\left(\prod_{i=1}^{\ell} \delta\left(\Gamma_{i}\right)\right) \rtimes \pi_{\text {cusp }}
\end{aligned}
$$

acts on $\pi^{(1)}$ by scalar $\epsilon_{\pi}((\rho, a))$ for $s=0$. From the condition $\operatorname{Jord}_{\rho}\left(\pi^{\prime}\right)=\emptyset$ we get that one needs to chose irreducible subrepresentation $\pi^{(1)}$ in a way that the normalized standard intertwining operator

$$
\begin{aligned}
\left(\prod_{i=1}^{\ell} \delta\left(\Gamma_{i}\right)\right) & \left.\times \delta\left(\left[\nu^{\left(a_{-}+1\right) / 2} \rho, \nu^{(a-1) / 2} \rho\right]\right)\right) \times \nu^{s} \delta\left(\rho, a_{-}\right) \rtimes \pi_{c u s p} \\
& \left.\rightarrow\left(\prod_{i=1}^{\ell} \delta\left(\Gamma_{i}\right)\right) \times \delta\left(\left[\nu^{\left(a_{-}+1\right) / 2} \rho, \nu^{(a-1) / 2} \rho\right]\right)\right) \times \nu^{-s} \delta\left(\rho, a_{-}\right) \rtimes \pi_{c u s p}
\end{aligned}
$$

acts on $\pi^{(1)}$ by scalar $\epsilon_{\pi}((\rho, a))$ for $s=0$.

From the other side, by (ii) of Lemma 3.5 the normalized standard intertwining operator

$$
\nu^{s} \delta\left(\rho, a_{-}\right) \rtimes \pi_{\text {cusp }} \rightarrow \nu^{-s} \delta\left(\rho, a_{-}\right) \rtimes \pi_{\text {cusp }}
$$

acts for $s=0$ on $T_{\tau_{\epsilon_{\pi}((\rho, a))}^{\left(\rho, \pi_{c u s p}\right)}}$ as $\epsilon_{\pi}((\rho, a))$. Therefore the normalized standard intertwining operator

$$
\left.\left(\prod_{i=1}^{\ell} \delta\left(\Gamma_{i}\right)\right) \times \delta\left(\left[\nu^{\left(a_{-}+1\right) / 2} \rho, \nu^{(a-1) / 2} \rho\right]\right)\right) \times \nu^{s} \delta\left(\rho, a_{-}\right) \rtimes \pi_{c u s p}
$$




$$
\left.\rightarrow\left(\prod_{i=1}^{\ell} \delta\left(\Gamma_{i}\right)\right) \times \delta\left(\left[\nu^{\left(a_{-}+1\right) / 2} \rho, \nu^{(a-1) / 2} \rho\right]\right)\right) \times \nu^{-s} \delta\left(\rho, a_{-}\right) \rtimes \pi_{c u s p}
$$

acts for $s=0$ as $\epsilon_{\pi}((\rho, a))$ on $\left.\left(\prod_{i=1}^{\ell} \delta\left(\Gamma_{i}\right)\right) \times \delta\left(\left[\nu^{\left(a_{-}+1\right) / 2} \rho, \nu^{(a-1) / 2} \rho\right]\right)\right) \rtimes T_{\tau_{\epsilon_{\pi}((\rho, a))}^{\left(\rho, \pi_{c u s p}\right)}}$.

From this we conclude

$$
\left.\pi^{(1)} \hookrightarrow\left(\prod_{i=1}^{\ell} \delta\left(\Gamma_{i}\right)\right) \times \delta\left(\left[\nu^{\left(a_{-}+1\right) / 2} \rho, \nu^{(a-1) / 2} \rho\right]\right)\right) \rtimes T_{\tau_{\epsilon_{\pi}((\rho, a))}^{\left(\rho, \pi_{c u s p}\right)}} .
$$

Very similarly as in the proof of Lemma 4.1 of $[\mathrm{MT}]$ we see that the representation on the right hand side of (4.6) has the unique irreducible subrepresentation (use also (3) of Lemma 3.2). Thus

$$
\pi^{(1)} \hookrightarrow\left(\prod_{i=1}^{\ell} \delta\left(\Gamma_{i}\right)\right) \rtimes \delta\left(\left[\nu^{-\left(a_{-}-1\right) / 2} \rho, \nu^{(a-1) / 2} \rho\right]_{T_{\tau_{\epsilon_{\pi}((\rho, a))}\left(\rho, \pi_{c u s p}\right)}} ; \pi_{c u s p}\right) .
$$

Now we know that for certain segments $\Gamma_{j}^{\prime}$ we have

$$
\pi \hookrightarrow\left(\prod_{j=1}^{\ell^{\prime}} \delta\left(\Gamma_{j}^{\prime}\right)\right) \times\left(\prod_{i=1}^{\ell} \delta\left(\Gamma_{i}\right)\right) \rtimes \delta\left(\left[\nu^{-\left(a_{-}-1\right) / 2} \rho, \nu^{(a-1) / 2} \rho_{T_{\tau_{\epsilon_{\pi}((\rho, a))}\left(\rho, \pi_{c u s p)}\right)}} ; \pi_{c u s p}\right)\right.
$$

(these segments depend on $\operatorname{Jord}(\pi)$; see $[\mathrm{M}]$ or $[\mathrm{MT}]$ for more information regarding them). Now Lemma 3.2 of $[\mathrm{MT}]$ implies that

$$
\pi \hookrightarrow \tau^{\prime} \rtimes \delta\left(\left[\nu^{-\left(a_{-}-1\right) / 2} \rho, \nu^{(a-1) / 2} \rho\right]_{T_{\left.\tau_{\epsilon_{\pi}\left(\rho, \pi_{c u s p}\right)}(\rho, a)\right)}} ; \pi_{\text {cusp }}\right) .
$$

for some irreducible representation $\tau^{\prime}$ of a general linear group. Further, (3.4) implies that

$$
\pi \hookrightarrow \tau^{\prime} \times \delta\left(\left[\nu \rho, \nu^{\left(a_{-}-1\right) / 2} \rho\right]\right) \rtimes \delta\left(\left[\rho, \nu^{(a-1) / 2} \rho\right]_{T_{\left.\tau_{\epsilon \pi}^{\left(\rho, \pi_{c u s p}\right.}(\rho, a)\right)}} ; \pi_{c u s p}\right) .
$$

Again Lemma 3.2 of [MT] implies that

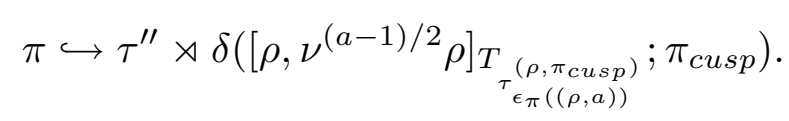

for some irreducible representation $\tau^{\prime \prime}$ of a general linear group. This implies that

$$
\pi \hookrightarrow \tau^{\prime \prime} \rtimes \delta\left(\left[\nu \rho, \nu^{(a-1) / 2} \rho\right]\right) \rtimes \tau_{\epsilon_{\pi}((\rho, a))}^{\left(\rho, \pi_{\text {cusp }}\right)} .
$$

Suppose now that

$$
\pi \hookrightarrow \tau^{\prime \prime \prime} \rtimes \delta\left(\left[\nu \rho, \nu^{(a-1) / 2} \rho\right]\right) \rtimes \tau_{-\epsilon_{\pi}((\rho, a))}^{\left(\rho, \pi_{\text {cusp }}\right)} .
$$


for some irreducible representation $\pi^{\prime \prime \prime}$ of a general linear group. Frobenius reciprocity implies that we must have a non-trivial intertwining from Jacquet module of $\pi$ into

$$
\tau^{\prime \prime \prime} \otimes \delta\left(\left[\nu \rho, \nu^{(a-1) / 2} \rho\right]\right) \rtimes \tau_{-\epsilon_{\pi}((\rho, a))}^{\left(\rho, \pi_{c u s p}\right)} .
$$

Since this representation has the unique irreducible subrepresentation

$$
\tau^{\prime \prime \prime} \otimes \delta\left(\left[\rho, \nu^{(a-1) / 2} \rho\right]_{\tau_{-\epsilon_{\pi}((\rho, a))}^{\left(\rho, \pi_{c u s p}\right)}} ; \pi_{c u s p}\right),
$$

we conclude that (4.15) is in the Jacquet module of $\pi$.

We shall now show that this is impossible. Actually, we shall show that (4.15) cannot be in Jacquet module of (4.8). From [T3] we know

$$
\mu^{*}\left(\delta\left(\left[\rho, \nu^{(a-1) / 2} \rho\right]_{\tau_{r}}, \sigma\right)\right)=\sum_{k=-1}^{(a-1) / 2} \delta\left(\left[\nu^{k+1} \rho, \nu^{(a-1) / 2} \rho\right]\right) \otimes \delta\left(\left[\rho, \nu^{k} \rho\right]_{\tau_{r}}, \sigma\right),
$$

where we take $\delta\left(\emptyset_{\tau_{r}}, \sigma\right)=\sigma$ and $\delta\left(\rho_{\tau_{r}}, \sigma\right)=\tau_{r}$ in the above formula.

To be able to get (4.15) in the Jacquet module of the right hand side of (4.11), we obviously must not take the term in the above sum corresponding to $k=(a-1) / 2$ since $\delta\left(\left[\rho, \nu^{(a-1) / 2} \rho\right]_{\tau_{r}}, \sigma\right) \not \delta\left(\left[\rho, \nu^{(a-1) / 2} \rho\right]_{\tau_{-r}}, \sigma\right)$. But for $k<(a-1) / 2$ we get $\nu^{(a-1) / 2} \rho$ on the left hand side of the tensor product $\otimes$ in (4.15). This is not possible since $a$ is maximal in $\operatorname{Jord}_{\rho}(\pi)$, and because of that, $\nu^{(a-1) / 2} \rho$ cannot be in the support of $\tau^{\prime \prime \prime}$. This proves the claim.

\section{REFERENCES}

[B] Ban, D., Parabolic induction and Jacquet modules of representations of $O(2 n, F)$, Glasnik Mat. 34(54) (1999), no. 3, 147-185.

[M] Mœglin, C., Sur la classification des séries discrètes des groupes classiques p-adiques: paramètres de Langlands et exhaustivité., Journal of the European Mathematical Society 4 (2002), no. 2, 143200 ..

[MT] Mœglin, C. and Tadić, M., Construction of discrete series for classical p-adic groups, J. Amer. Math. Soc. 15 (2002), 715-786.

[MVW] Møglin, C.; Vignéras, M.-F. and Waldspurger, J.-L., Correspondances de Howe sur un corps p-adique, Lecture Notes in Math. 1291, Springer-Verlag, Berlin, 1987.

[S] Silberger, A., Special representations of reductive p-adic groups are not integrable, Ann. of Math. 111 (1980), 571-587.

[T1] Tadić, M., Structure arising from induction and Jacquet modules of representations of classical p-adic groups, Journal of Algebra 177 (1995), no. 1, 1-33.

[T2] Tadić, M., On reducibility of parabolic induction, Israel J. Math. 107 (1998), 29-91.

[T3] Tadić, M. Square integrable representations of classical $p$-adic groups corresponding to segments, Representation Theory 3 (1999), 58-89.

[T4] Tadić, M., On classification of some classes of irreducible representations of classical groups, Representations of real and $p$-adic groups, Singapore University Press and World Scientific, Singapore, 2004, pp. 95-162.

[Z] Zelevinsky, A. V.:, Induced representations of reductive p-adic groups II. On irreducible representations of $G L(n)$, Ann. Sci. École Norm. Sup. 13 (1980), 165-210.

Department of Mathematics, University of Zagreb, Bijenička 30, 10000 Zagreb, Croatia

E-mail address: tadic@math.hr 